Discrete Comput Geom 31:251-255 (2004)

DOI: $10.1007 / \mathrm{s} 00454-003-2859-\mathrm{z}$

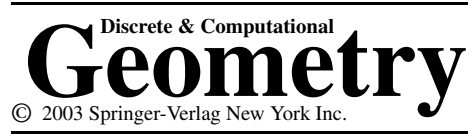

\title{
Bounded VC-Dimension Implies a Fractional Helly Theorem
}

\author{
Jiří Matoušek* \\ Department of Applied Mathematics and \\ Institute of Theoretical Computer Science (ITI), Charles University, \\ Malostranské nám. 25, 11800 Praha 1, Czech Republic \\ matousek@kam.mff.cuni.cz
}

\begin{abstract}
We prove that every set system of bounded VC-dimension has a fractional Helly property. More precisely, if the dual shatter function of a set system $\mathcal{F}$ is bounded by $o\left(m^{k}\right)$, then $\mathcal{F}$ has fractional Helly number $k$. This means that for every $\alpha>0$ there exists a $\beta>0$ such that if $F_{1}, F_{2}, \ldots, F_{n} \in \mathcal{F}$ are sets with $\bigcap_{i \in I} F_{i} \neq \emptyset$ for at least $\alpha\left(\begin{array}{l}n \\ k\end{array}\right)$ sets $I \subseteq\{1,2, \ldots, n\}$ of size $k$, then there exists a point common to at least $\beta n$ of the $F_{i}$. This further implies a $(p, k)$-theorem: for every $\mathcal{F}$ as above and every $p \geq k$ there exists $T$ such that if $\mathcal{G} \subseteq \mathcal{F}$ is a finite subfamily where among every $p$ sets, some $k$ intersect, then $\mathcal{G}$ has a transversal of size $T$. The assumption about bounded dual shatter function applies, for example, to families of sets in $\mathbf{R}^{d}$ definable by a bounded number of polynomial inequalities of bounded degree; in this case we obtain fractional Helly number $d+1$.
\end{abstract}

\section{Introduction}

The well-known theorem of Helly states that if $\mathcal{C}$ is a finite family of convex sets in $\mathbf{R}^{d}$ such that any $d+1$ or fewer of the sets of $\mathcal{F}$ intersect, then $\bigcap \mathcal{C} \neq \emptyset$; we say that the $d$-dimensional convex sets have Helly number $d+1$. A vast number of Helly-type results are known; see, e.g., [8].

Here we consider fractional Helly-type theorems. We introduce them briefly; they are discussed more leisurely in [12], together with other topics of this paper, such as VC-dimension and $(p, q)$-theorems.

The original fractional Helly theorem for convex sets in $\mathbf{R}^{d}$, asserts the following (here and in what follows, we use the notation $[n]=\{1,2, \ldots, n\}$ and $\left(\begin{array}{l}X \\ k\end{array}\right)$ for the system of all $k$-element subsets of $X$ ):

\footnotetext{
* Supported by Project LN00A056 of the Ministry of Education of the Czech Republic. Part of this research was done during a visit to Technion in Haifa.
} 
Theorem $1[10] . \quad$ For every $d \geq 1$ and every $\alpha \in(0,1]$ there exists a $\beta=\beta(d, \alpha)>0$ with the following property. Let $C_{1}, \ldots, C_{n}$ be convex sets in $\mathbf{R}^{d}$ such that $\bigcap_{i \in I} C_{i} \neq \emptyset$ for at least $\alpha\left(\begin{array}{c}n \\ d+1\end{array}\right)$ index sets $I \in\left(\begin{array}{c}{[n]} \\ d+1\end{array}\right)$. Then there exists a point contained in at least $\beta n$ of the $C_{i}$.

Let $\mathcal{F}$ be an arbitrary set system. For sets $F_{1}, F_{2}, \ldots, F_{n} \in \mathcal{F}$ and an index set $I \subseteq[n]$, we write $F_{I}$ for $\bigcap_{i \in I} F_{i}$. We say that $\mathcal{F}$ has fractional Helly number $k$ if for every $\alpha>0$ there exists a $\beta>0$ such that if $n$ is any natural number and $F_{1}, F_{2}, \ldots, F_{n} \in \mathcal{F}$ are sets such that $F_{I} \neq \emptyset$ for at least $\alpha\left(\begin{array}{l}n \\ k\end{array}\right)$ sets $I \in\left(\begin{array}{c}{[n]} \\ k\end{array}\right)$, then there exists a point common to at least $\beta n$ of the $F_{i}$. We say that $\mathcal{F}$ has the fractional Helly property if it has a finite fractional Helly number.

Note that this definition formally makes sense only for infinite set systems $\mathcal{F}$; if $\mathcal{F}$ is finite, then the fractional Helly number is trivially 1 , since $\beta$ can be chosen in dependence on the number of sets in $\mathcal{F}$. However, in concrete examples, we usually also have an explicit dependence of $\beta$ on $\alpha$, and so we can make conclusions about finite set systems too.

Although the fractional Helly property appears less intuitive than the Helly property, and its conclusion is weaker, it seems much better behaved and more robust in general than the Helly property. Here are some examples:

- There is a fractional Helly theorem for hyperplane transversals of convex sets in $\mathbf{R}^{d}[1]$ although there is no finite Helly number.

- For convex lattice sets in $\mathbf{Z}^{d}$ (i.e., intersections of convex sets in $\mathbf{R}^{d}$ with the $d$ dimensional integer lattice), the Helly number is $2^{d}$, anomalously large, but the fractional Helly number is only $d+1$ [4].

- If a family $\mathcal{F}$ has fractional Helly number $k$ then the family $\left\{F_{1} \cup F_{2}: F_{1}, F_{2} \in \mathcal{F}\right\}$, too, has fractional Helly number $k$, as is easily checked; for the Helly number this, of course, fails badly.

In this paper we further support the above thesis by adding a wide class of examples with the fractional Helly property: all set systems of bounded VC-dimension.

The VC-dimension of a set system $\mathcal{F}$ on a ground set $X$ is the maximum size of a set $A \subseteq X$ that is shattered by $\mathcal{F}$, meaning that $\{A \cap F: F \in \mathcal{F}\}=2^{A}$. Examples of set systems with bounded VC-dimension abound in geometry; see, e.g., [11] for a wider background. The dual shatter function of $\mathcal{F}$ is a function $\pi_{\mathcal{F}}^{*}: \mathbf{N} \rightarrow \mathbf{N}$, and $\pi_{\mathcal{F}}^{*}(m)$ is the maximum number of nonempty fields of the Venn diagram of $m$ sets of $\mathcal{F}$. More formally, we call two points $x, y \in X$ equivalent with respect to sets $F_{1}, \ldots, F_{m}$ if $\left\{i \in[m]: x \in F_{i}\right\}=\left\{i \in[m]: y \in F_{i}\right\}$, and $\pi_{\mathcal{F}}^{*}(m)$ is the maximum possible number of classes of this equivalence over all choices of $F_{1}, \ldots, F_{m} \in \mathcal{F}$. The dual $V C$-dimension of $\mathcal{F}$ is the maximum possible number of sets in $\mathcal{F}$ with a complete Venn diagram, i.e., $\max \left\{k: \pi_{\mathcal{F}}^{*}(k)=2^{k}\right\}$. It is well known that if the dual VC-dimension is $d^{*}$, then $\pi_{\mathcal{F}}^{*}(m) \leq \sum_{i=0}^{d^{*}}\left(\begin{array}{c}m \\ i\end{array}\right)$. Moreover, $d^{*} \leq 2^{d}$, where $d$ is the VC-dimension, and, in particular, the VC-dimension is finite iff the dual VC-dimension is.

The dual shatter function seems to be a crucial quantitative parameter of geometric set systems; for example, it is relevant to the performance of range-searching data structures [7], and in many cases it essentially determines the discrepancy of the set system [11]. 
The following theorem shows a similar phenomenon for the fractional Helly number.

Theorem 2 (Fractional Helly for Bounded VC-Dimension). Let $\mathcal{F}$ be a set system whose dual shatter function satisfies $\pi_{\mathcal{F}}^{*}(m)=o\left(m^{k}\right)$ (that is, $\left.\lim _{m \rightarrow \infty} \pi_{\mathcal{F}}^{*}(m) / m^{k}=0\right)$, where $k$ is a fixed integer (in particular, this holds if the dual VC-dimension of $\mathcal{F}$ is at most $k-1)$. Then $\mathcal{F}$ has fractional Helly number $k$.

In contrast, bounded VC-dimension does not guarantee any Helly property. A very simple example is the system $\{[n] \backslash\{i\}: i \in[n]\}$, and more complicated examples will be mentioned later.

We note that the original Katchalski-Liu theorem (Theorem 1) is not a special case of Theorem 2, since convex sets in $\mathbf{R}^{d}$ have infinite VC-dimension.

A primary example of geometric families of bounded VC-dimension are semialgebraic sets in $\mathbf{R}^{d}$ of bounded description complexity. We recall that a set $A \subseteq \mathbf{R}^{d}$ is semialgebraic if it can be defined by a Boolean combination of polynomial inequalities; that is, if $A=\left\{x \in \mathbf{R}^{d}: \Phi\left(p_{1}(x) \geq 0, p_{2}(x) \geq 0, p_{r}(x) \geq 0\right)\right\}$, where $\Phi$ is a Boolean formula and $p_{1}, \ldots, p_{r} \in \mathbf{R}\left[x_{1}, \ldots, x_{d}\right]$ are polynomials. (The definition of a semialgebraic set may also involve quantifiers. However, by a well-known result of Tarski, quantifiers can be eliminated, and so each such set has an equivalent quantifier-free definition; see, e.g., [6] for a discussion of semialgebraic sets and quantifier elimination.) We call the number $\max (d, r, D)$, where $D$ is the maximum degree of the $p_{i}$, the description complexity of $A$. Standard estimates on the number of sign patterns of real polynomials (due to Oleinik, Petrovskii, Milnor, Thom; see, e.g., [5] for precise results and references) imply that if $\mathcal{F}$ is the family of all semialgebraic sets in $\mathbf{R}^{d}$ of description complexity at most $B$, then $\pi_{\mathcal{F}}^{*}(m) \leq C m^{d}$ for some $C=C(B)$ and all $m$. More generally, if $\mathcal{F}$ is as before and $\mathcal{F}^{\prime}=\{F \cap V: F \in \mathcal{F}\}$, where $V$ is a $k$-dimensional algebraic variety in $\mathbf{R}^{d}$, then $\pi_{\mathcal{F}^{\prime}}^{*}(m) \leq C^{\prime} m^{k}, C^{\prime}=C^{\prime}(B, k)$ [5]. We thus have:

Corollary 3. For every fixed B, the family of all semialgebraic subsets of $\mathbf{R}^{d}$ of description complexity at most $B$ has fractional Helly number $d+1$. The system of all intersections of sets of this family with a fixed $k$-dimensional algebraic variety has fractional Helly number $k+1$.

Here is a nice more concrete example. If $F \subseteq \mathbf{R}^{d}$ is a semialgebraic set of bounded description complexity, then the set of all $j$-flats in $\mathbf{R}^{d}$ intersecting $F$ can be represented by a semialgebraic subset of the affine Grassmannian, which is a $(j+1)(d-j)$-dimensional algebraic variety. Consequently, there is a fractional Helly theorem: If $\mathcal{F}$ is the family all semialgebraic subsets of $\mathbf{R}^{d}$ of description complexity at most $B, F_{1}, \ldots, F_{n} \in \mathcal{F}$, and at least $\alpha\left(\begin{array}{l}n \\ k\end{array}\right)$ of the $k$-tuples of the $F_{i}$ have a $j$-flat transversal, where $k=(j+1)(d-j)$, then there is a $j$-flat intersecting at least $\beta n$ of the $F_{i}$. In particular, for line transversals for semialgebraic sets of bounded description complexity in $\mathbf{R}^{3}$ we obtain the fractional Helly number 5. (In contrast, there is no fractional Helly theorem for line transversals of convex sets in $\mathbf{R}^{3}$.)

Alon and Kleitman [3] established an old conjecture of Hadwiger and Debrunner, the ( $p, q)$-theorem for convex sets: For every integer $d, p, q, p \geq q \geq d+1$, there exists $T$ such that whenever $\mathcal{F}$ is a finite family of convex sets in $\mathbf{R}^{d}$ such that among every $p$ 
sets of $\mathcal{F}$, some q intersect, then $\tau(\mathcal{F}) \geq T$; that is, there is a $T$-point set intersecting all sets of $\mathcal{F}$. Their spectacular proof uses the Katchalski-Liu fractional Helly theorem in an essential way. As discussed in [2], their method can be used to derive $(p, q)$-theorems from fractional Helly theorems on a fairly abstract level. These methods immediately imply that a family $\mathcal{F}$ as in Theorem 2 satisfies a $(p, k)$-theorem (for every $p \geq k$ ):

Theorem $4((p, q)$-Theorem for Bounded VC-Dimension). Let $\mathcal{F}$ be a set system with $\pi_{\mathcal{F}}^{*}(m)=o\left(m^{k}\right)$ for some integer $k$, and let $p \geq k$. Then there is a constant $T$ such that the following holds for every finite family $\mathcal{G} \subseteq \mathcal{F}:$ If $\mathcal{G}$ has the ( $p, k)$-property, meaning that among every $p$ sets of $\mathcal{F}$, some $k$ intersect, then $\tau(\mathcal{G}) \leq T$.

The Alon-Kleitman method is explained in many sources [3], [12], [2], [4], and so we omit a detailed discussion. However, for readers familiar with the method, we remark that the first step (showing that the fractional packing number of $\mathcal{G}$ is bounded) goes through unchanged based on the fractional Helly property, as well as the second step (LP duality), and the third step ( $\varepsilon$-net property, or bounding $\tau$ in terms of $\tau^{*}$ ) is just the well-known theorem of Haussler and Welzl [9] about the existence of $\varepsilon$-nets for systems of bounded VC-dimension.

Already the case $p=k$ in Theorem 4 is interesting and appears nontrivial. It shows that while a set system of bounded VC-dimension may fail to have a Helly property, there is always an "almost-Helly theorem" (a Gallai-type theorem according to common terminology): If every $k$ sets intersect, then all sets can be intersected by a bounded number of points.

\section{Proof of Theorem 2}

Let $\mathcal{F}$ and $k$ be as in Theorem 2 , let $\alpha>0$ be given, and let $F_{1}, F_{2}, \ldots, F_{n} \in \mathcal{F}$ be sets such that $F_{I} \neq \emptyset$ for at least $\alpha\left(\begin{array}{c}n \\ k\end{array}\right) k$-tuples $I \in\left(\begin{array}{c}{[n]} \\ k\end{array}\right)$. We may assume that $n$ is larger than any given constant, for otherwise, for $\beta$ sufficiently small, it is enough to have a point in a single $F_{i}$.

Using the assumption $\pi_{\mathcal{F}}^{*}(m)=o\left(m^{k}\right)$, we fix $m$ so that $\pi_{\mathcal{F}}^{*}(m)<\frac{1}{4} \alpha\left(\begin{array}{c}m \\ k\end{array}\right)$, and we set $\beta=1 / 2 m$. Finally, we assume that $n$ is so large that $\beta n \geq m$.

For contradiction, we suppose that no point is common to $\beta n$ of the $F_{i}$. We consider

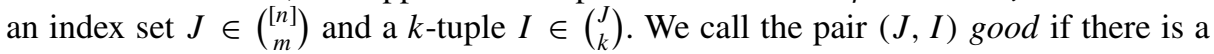
point $x$ with $x \in F_{i}$ for all $i \in I$ and $x \notin F_{j}$ for all $j \in J \backslash I$. We bound below the probability that a pair $(J, I)$ chosen uniformly at random is good.

We first choose a random $I \in\left(\begin{array}{c}{[n]} \\ k\end{array}\right)$, and then we choose the $m-k$ elements of $J \backslash I$ at random from $[n] \backslash I$. The probability that $F_{I} \neq \emptyset$ is at least $\alpha$. If $F_{I} \neq \emptyset$, we fix one point $x \in F_{I}$. By the assumption, $x$ is contained in fewer than $\beta n$ of the $F_{i}$, and so the probability that none of the sets $F_{j}$ with $j \in J \backslash I$ contains $x$ is at least

$$
\frac{\left(\begin{array}{c}
(1-\beta) n\rceil \\
m-k
\end{array}\right)}{\left(\begin{array}{c}
n-k \\
m-k
\end{array}\right)} \geq \prod_{i=0}^{m-k-1} \frac{(1-\beta) n-i}{n-i} \geq\left(\frac{(1-\beta) n-m}{n-m}\right)^{m} .
$$


Since we assumed $m \leq \beta n$ and $\beta=1 / 2 m$, the above expression is at least $(1-2 \beta)^{m}=$ $(1-1 / m)^{m} \geq \frac{1}{4}$. Therefore, the probability of a random pair $(J, I)$ being good is at least $\frac{1}{4} \alpha$.

If we choose a random $J \in\left(\begin{array}{c}{[n]} \\ m\end{array}\right)$, the expected number of $I \in\left(\begin{array}{l}J \\ k\end{array}\right)$ with $(J, I)$ good is at least $N=\frac{1}{4} \alpha\left(\begin{array}{c}m \\ k\end{array}\right)$, and so there exists a $J$ with at least this many $I$. However, this violates the assumption $\pi_{\mathcal{F}}^{*}(m)<N$, since the sets indexed by $J$ have at least $N$ nonempty fields in their Venn diagram.

\section{Acknowledgments}

I thank Roy Meshulam and Meir Katchalski for inspiring discussions. I also thank the referee for helpful comments.

\section{References}

1. N. Alon and G. Kalai. Bounding the piercing number. Discrete Comput. Geom., 13:245-256, 1995.

2. N. Alon, G. Kalai, J. Matoušek, and R. Meshulam. Transversal numbers for hypergraphs arising in geometry. Adv. Appl. Math., 130:2509-2514, 2002.

3. N. Alon and D. Kleitman. Piercing convex sets and the Hadwiger-Debrunner $(p, q)$-problem. Adv. in Math., 96(1):103-112, 1992.

4. I. Bárány and J. Matoušek. A fractional Helly theorem for convex lattice sets. Adv. in Math., 174:227-235, 2003.

5. S. Basu, R. Pollack, and M.-F. Roy. On the number of cells defined by a family of polynomials on a variety. Mathematika, 43:120-126, 1996.

6. J. Bochnak, M. Coste, and M.-F. Roy. Real Algebraic Geometry. Springer-Verlag, Berlin, 1998. Transl. from the French, revised and updated edition.

7. B. Chazelle and E. Welzl. Quasi-optimal range searching in spaces of finite VC-dimension. Discrete Comput. Geom., 4:467-489, 1989.

8. J. Eckhoff. Helly, Radon and Carathéodory type theorems. In P. M. Gruber and J. M. Wills, editors, Handbook of Convex Geometry, pages 389-448. North-Holland, Amsterdam, 1993.

9. D. Haussler and E. Welzl. Epsilon-nets and simplex range queries. Discrete Comput. Geom., 2:127-151, 1987.

10. M. Katchalski and A. Liu. A problem of geometry in $R^{n}$. Proc. Amer. Math. Soc., 75:284-288, 1979.

11. J. Matoušek. Geometric Discrepancy (An Illustrated Guide). Springer-Verlag, Berlin, 1999.

12. J. Matoušek. Lectures on Discrete Geometry. Springer-Verlag, New York, 2002.

Received March 24, 2002, and in revised form April 4, 2003. Online publication December 31, 2003. 